\title{
Functional characterization of genes with circadian expression patterns in common wheat
}

\author{
Kiseleva A.A.*, Bragina M.K., Salina E.A. \\ Institute of Cytology and Genetics, SB RAS, Novosibirsk, Russia \\ *email: antkiseleva@bionet.nsc.ru
}

The periodicity inherent for such factors, as light and temperature, is expressed in most physiological processes and the behavior of most living organisms. In wheat some key components of plant circadian rhythms have been identified, but there is little data on their daily expression and interaction of these genes. During the project, using the systemic analysis of the wheat transcriptome, genes expressed in the circadian type and the metabolic pathways controlled by them were identified: responses to stimuli and nutrients, transport, photoperiodism, photomorphogenesis, synthesis and degradation of different metabolites, regulation of the processes of RNA synthesis. It was shown that a significant part of the transcriptome is under the control of circadian rhythms, and the expression of these genes can vary greatly with time. We identified five expression patterns characterized by peaks at different time points and described the genes underlying these patterns. We analyzed the enrichment of gene ontology terms with various patterns and described the main metabolic pathways in each group. Transcription factor enrichment analysis of genes in different modules shown that TCP transcription factors are common regulators for the circadian genes. The specific transcription factors for every module were also detected. We identified homologues of the genes of circadian rhythms in Arabidopsis, assessed and compared the patterns of their expression. For the majority of genes, the patterns coincided, which may indicate the conservatism of genes for circadian rhythms in common wheat relative to the Arabidopsis.

Acknowledgements: The reported study was funded by RFBR, project number 20-31680003. 